\title{
Comparison of Flow-Controlled Calcium and Barium Carbonate Precipitation
} Patterns

\author{
G. Schuszter ${ }^{1}$ and A. De Wit ${ }^{1, a)}$ \\ Université libre de Bruxelles (ULB), Nonlinear Physical Chemistry Unit, \\ CP231, 1050 Brussels, Belgium
}

(Dated: 21 December 2016)

Various precipitation patterns can be obtained in flow conditions when injecting a solution of sodium carbonate in a confined geometry initially filled with a solution of either barium or calcium chloride. We compare here the barium and calcium carbonate precipitate structures obtained as a function of initial concentrations and injection flow rate. We show that, in some part of the parameter space, the patterns are similar and feature comparative properties indicating that barium and calcium behave similarly in the related flow-controlled precipitation conditions. For other values of parameters though, the precipitate structures are different indicating that the cohesive and microscopic properties of barium versus calcium carbonate are then important in shaping the pattern in flow conditions.

PACS numbers: 47.70.Fw Chemically reactive flows; 47.15.gp Hele-Shaw flows; 82.40.Ck Pattern formation in reactions with diffusion, flow and heat transfer Keywords: flow-driven precipitation; pattern formation; $\mathrm{CO}_{2}$ mineralization; carbonate.

a) To whom the correspondence should be addressed. Email: adewit@ulb.ac.be. Phone: +32 26505774 . 


\section{INTRODUCTION}

The physical and chemical properties of a material are not only determined by its chemical composition but also by its morphology at both micro- and macroscale. In that respect, it has recently been shown that out-of-equilibrium conditions can be used to control and shape solid phases at both micro- and macroscales ${ }^{1-7}$. Specifically, innovative growth conditions of materials can be obtained by conducting precipitation reactions within gradients of concentrations controlled by hydrodynamic fluxes. Such gradients provide additional thermodynamic forces compared to a classical chemical synthesis in which the reactants are thoroughly mixed to maximize the rate of reaction. These out-of-equilibrium forces generate conditions that can favor, for example, the production of thermodynamically unstable crystalline forms $^{3}$, or lead to microstuctures and compositions different from those obtained in homogeneous systems ${ }^{8-11}$.

Our objective here is to analyze to what extent such flow-conditions can produce similar macroscopic patterns for barium or calcium carbonate precipitates obtained when injecting an aqueous solution of sodium carbonate in a confined geometry initially filled with an aqueous solution of either barium or calcium chloride. We focus on such reactants for various reasons. First, mineralization of carbon dioxide in soils is attracting increased interest in the framework of $\mathrm{CO}_{2}$ sequestration aiming at reducing the atmospheric concentration of this greenhouse gas ${ }^{12,13}$. In this case, carbonates resulting from $\mathrm{CO}_{2}$ dissolution in water can react with minerals like $\mathrm{Ca}^{2+}$ or $\mathrm{Mg}^{2+}$ to yield solid precipitates, which increases the safety of the sequestration process. It has recently been shown that such a mineralization can be responsible for a fast consumption of $\mathrm{CO}_{2}$ upon its injection in soils ${ }^{13}$. The complex chemistry of $\mathrm{CO}_{2}$ in supercritical conditions has also an influence on pore water chemistry including that of Ba-bearing minerals ${ }^{14}$. Moreover, as in situ experiments on real geological formations are difficult to do, it is of interest to perform laboratory studies of calcium carbonate mineralization in real reservoir samples ${ }^{15}$. NMR or X-ray data can then give access to the 3D structure of the solid carbonate precipitates. Thanks to an enhanced contrast of barium with regard to calcium in X-ray analysis ${ }^{16}$, it is of interest to understand whether the spatial distribution and amount of barium carbonate precipitates is similar or not to the calcium carbonate ones in simple 2D geometry to assess whether X-ray studies based on barium could be representative of those with calcium or not. This would allow 
to conduct precipitation studies in 3D opaque systems with a larger sensitivity giving both liquid dynamics and solid distribution using $\mathrm{Ba}^{2+}$ solutions.

In parallel, barium carbonates also have applications in cement ${ }^{17,18}$ and carbon capture and utilization ${ }^{19}$ technologies. There is thus also interest to understand how their precipitation patterns vary depending on flow conditions. We have recently shown using chemical garden recipes ${ }^{20}$ that the macroscopic morphology of flow-driven precipitation patterns can be robust with regard to changes in reactants even though differences can appear depending on the cohesion of the solid phase. Meanwhile, we have also developed various tools to quantitatively assess the macroscopic properties of such patterns ${ }^{2,21}$. Our goal is to use such quantitative measures to analyze the robustness of the change of calcium to barium in the amount and spatial distribution of their carbonate precipitates when produced in flow conditions.

In this context, we study here experimentally the properties of barium and calcium carbonates produced in a Hele-Shaw cell (quasi 2D confined geometry) when carbonate ions are injected into a solution of either $\mathrm{Ba}^{2+}$ or $\mathrm{Ca}^{2+}$ at a constant flow rate. We show that in some parameter range spanned by the flow rate and initial reactant concentrations, the barium or calcium carbonate patterns are similar while they differ for other values of the parameters. These differences are quantified by measuring quantitative properties like the total grayscale intensity, the filling, and the density of the patterns.

This study shows that barium and calcium behave in some cases similarly to produce the same type of carbonate precipitates in flow conditions while they can produce quite different solid distributions in other cases. This highlights the interest of flow-driven conditions to control the output of precipitation reactions and defines the conditions in which $\mathrm{Ba}^{2+}$ could be used as an alternative to $\mathrm{Ca}^{2+}$ for studies of carbonate precipitates.

\section{EXPERIMENTAL}

The flow-driven precipitation experiments are performed in a horizontal confined geometry (Hele-Shaw cell) maintained between two parallel Plexiglas plates vertically separated by a $0.5 \mathrm{~mm}$ thick gap. The experimental setup is identical to the one used in our previous studies of calcium carbonate precipitation patterns ${ }^{2,21}$. To produce precipitates, an aqueous solution of sodium carbonate, $\mathrm{Na}_{2} \mathrm{CO}_{3}$, is radially injected from below with a syringe 
TABLE I. Molar concentration, $c(\mathrm{~mol} / \mathrm{L})$, normalized concentration, $[X]_{n}$ (defined using the solubility in water $c_{\max }=6.67 \mathrm{~mol} / \mathrm{L}$ for $\mathrm{CaCl}_{2}, c_{\max }=1.72 \mathrm{~mol} / \mathrm{L}$ for $\mathrm{BaCl}_{2}$, and $c_{\max }=$ $2.90 \mathrm{~mol} / \mathrm{L}$ for $\left.\mathrm{Na}_{2} \mathrm{CO}_{3}\right)$, density, $\rho(\mathrm{kg} / \mathrm{L})$, and dynamic viscosity, $\mu$ ( $\left.\mathrm{mPa} \mathrm{s}\right)$, of the solutions at $T=(21 \pm 1)^{\circ} \mathrm{C}$.

\begin{tabular}{l|cccc} 
Chemical & $c$ & {$[X]_{n}$} & $\rho$ & $\mu$ \\
\hline $\mathrm{CaCl}_{2}$ & 0.50 & 0.08 & 1.043 & 1.18 \\
& 1.50 & 0.23 & 1.128 & 1.61 \\
& 4.50 & 0.68 & 1.360 & 5.99 \\
\hline $\mathrm{BaCl}_{2}$ & 0.13 & 0.08 & 1.021 & 1.08 \\
& 0.37 & 0.23 & 1.064 & 1.17 \\
& 1.16 & 0.68 & 1.203 & 1.36 \\
\hline $\mathrm{Na}_{2} \mathrm{CO}_{3}$ & 0.25 & 0.09 & 1.023 & 1.19 \\
& 0.75 & 0.26 & 1.072 & 1.48 \\
& 1.50 & 0.52 & 1.141 & 2.13
\end{tabular}

pump through a tiny inlet into the gap filled either with a calcium chloride, $\mathrm{CaCl}_{2}$, or a barium chloride, $\mathrm{BaCl}_{2}$, solution. When the reactants get into contact, a white precipitate,

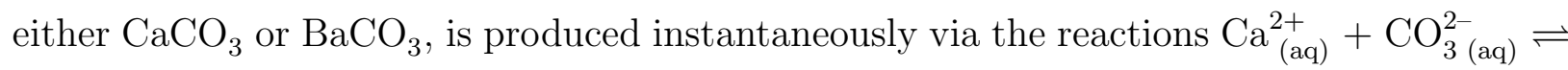
$\mathrm{CaCO}_{3(\mathrm{~s})}$ or $\mathrm{Ba}_{(\mathrm{aq})}^{2+}+\mathrm{CO}_{3(\text { aq })}^{2-} \rightleftharpoons \mathrm{BaCO}_{3(\mathrm{~s})}$. The precipitation pattern growing during the injection is monitored by a digital camera from above. In each experiment, $3 \mathrm{~mL}$ of $\mathrm{Na}_{2} \mathrm{CO}_{3}$ solution is injected at various constant volumetric flow rates $(Q=0.1,1.0$, and $6.5 \mathrm{~mL} / \mathrm{min})$. The $\mathrm{pH}$ of the $\mathrm{Na}_{2} \mathrm{CO}_{3}$ solution is adjusted to 10 to avoid the production of calcium hydroxide and barium hydroxide precipitates. The normalized concentrations of reactants, $[X]_{n}=c_{x} / c_{\max }$, which are their dimensional concentrations, $c_{x}$, divided by their solubility in water, $c_{\max }$, are also varied from one experiment to the other. The density and viscosity of the reactant solutions are measured at the temperature of the experiments using an Anton Paar DMA 35 densitometer and a Brookfield DV-II + Pro Extra viscosimeter respectively (see Table I). 

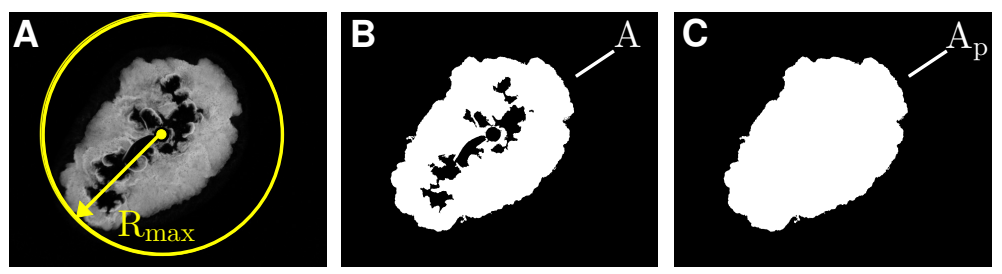

FIG. 1. Radius $R_{\max }$ of the circle drawn around the precipitation pattern (A), area $A$ covered by the precipitate $(\mathrm{B})$, and area $A_{\mathrm{p}}$ of the region inside the pattern perimeter $(\mathrm{C})$ for one particular pattern.

\section{PATTERN FORMATION AND CHARACTERIZATION}

When the injection starts, the reactants begin to mix and a precipitate appears. A large variety of precipitation patterns has been studied recently in the $\mathrm{CaCO}_{3}$ system in a similar confined geometry ${ }^{2,21}$. The patterns have been characterized from a $\mathrm{CO}_{2}$ sequestration efficiency point of view ${ }^{21}$. In the present work, our objective is to compare precipitation patterns obtained for various experimental conditions using the $\mathrm{Ca}^{2+}-\mathrm{CO}_{3}^{2-}$ and $\mathrm{Ba}^{2+}-$ $\mathrm{CO}_{3}^{2-}$ reactant pairs. To do so, we determine some characteristic quantities of the patterns as introduced previously ${ }^{2,21}$ : The amount of precipitate produced can be qualitatively measured by the total grayscale intensity, $I_{\text {tot }}(t)=\sum_{x, y} I_{n}(x, y, t)$, of the image taken at time $t$, where $I_{n}(x, y, t)$ is the grayscale value normalized by the background grayscale intensity, and $x$ and $y$ are spatial coordinates.

To characterize the spatial distribution of the precipitation patterns, we also measure 3 quantities: (I) the radius $R_{\max }$ being the largest distance between the inlet and the pattern perimeter (Fig. 1A); (II) the area $A$ covered by precipitate particles (Fig. 1B); and (III) the area $A_{\mathrm{p}}$ of the region inside the pattern perimeter (Fig. 1C). On the basis of those quantities, we next compute the filling $F=A / A_{\mathrm{p}} \in[0,1]$, measuring whether the precipitate entirely covers the region inside the pattern perimeter $(F=1)$ or not $(F<1)$. For an essentially hollow pattern, $F \ll 1$. We also compute the pattern density, $d=A_{\mathrm{p}} /\left(\pi R_{\max }^{2}\right) \in[0,1]$, comparing the area $A_{\mathrm{p}}$ of the pattern to the area of the circle of radius $R_{\max }$. A large value of $d$ indicates a circularly spreading pattern while a low value of $d$ corresponds to a pattern with a few preferred growth directions.

The patterns obtained with either barium or calcium ions may be compared to each other visually and using the quantities $I_{\text {tot }}, F$, and $d$ to investigate whether replacing one reactant 


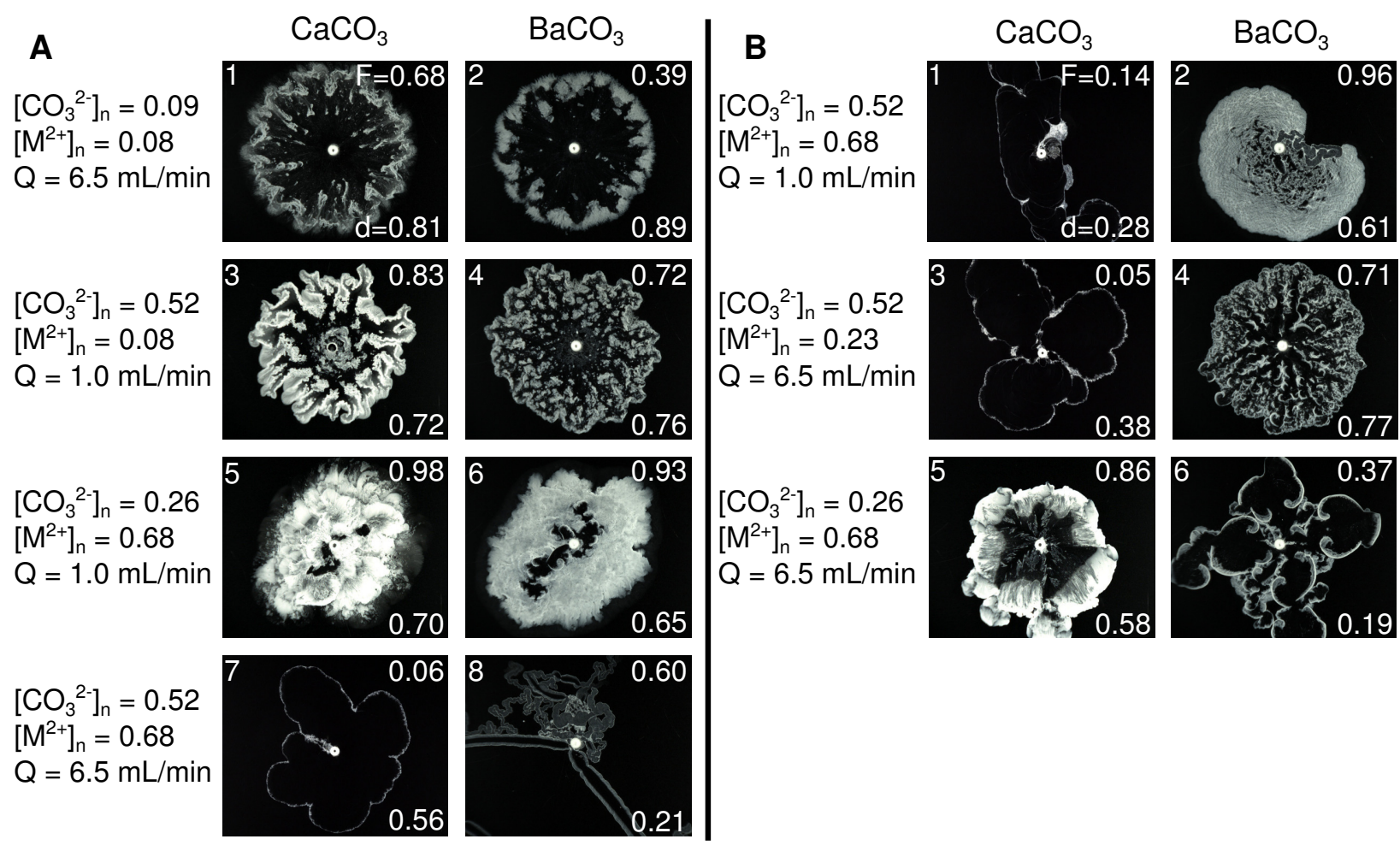

FIG. 2. Characteristic similarities (A) and major differences (B) between $\mathrm{CaCO}_{3}$ and $\mathrm{BaCO}_{3}$ precipitation patterns observed for various experimental conditions. $\left[\mathrm{M}^{2+}\right]_{\mathrm{n}}$ refers to the normalized concentration of either the $\mathrm{Ca}^{2+}$ or the $\mathrm{Ba}^{2+}$ reactant solution and $Q$ is the flow rate. The numbers in the top left, top right, and bottom right corners of the images are the image number, the filling $F$, and the pattern density $d$, respectively. Field of view: $123 \mathrm{~mm} \times 98 \mathrm{~mm}$.

by the other one will significantly modify the pattern formation or not.

\section{RESULTS}

To investigate whether the precipitation patterns obtained recently in flow conditions for the $\mathrm{CaCO}_{3}$ system ${ }^{2,21}$ are similar to those obtained with $\mathrm{BaCO}_{3}$, we have performed experiments with $\mathrm{Ba}^{2+}$ solutions having exactly the same normalized concentrations as the $\mathrm{Ca}^{2+}$ solutions (Table I). A selection of patterns is shown in Fig. 2 highlighting remarkable similarities (panel A) but also major differences (panel B) depending on the values of parameters.

Let us first note that for low flow rates and low reactant concentrations, small separated precipitate particles are produced in both systems as shown in Fig. 3. Those particles have 


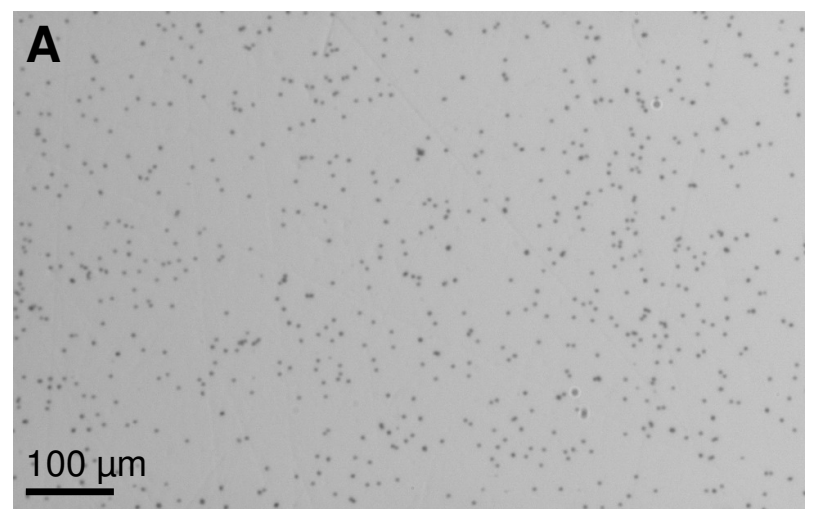

$\left[\mathrm{Na}_{2} \mathrm{CO}_{3}\right]_{\mathrm{n}}=0.07 \quad\left[\mathrm{CaCl}_{2}\right]_{\mathrm{n}}=0.03$

$\mathrm{Q}=0.1 \mathrm{~mL} / \mathrm{min}$

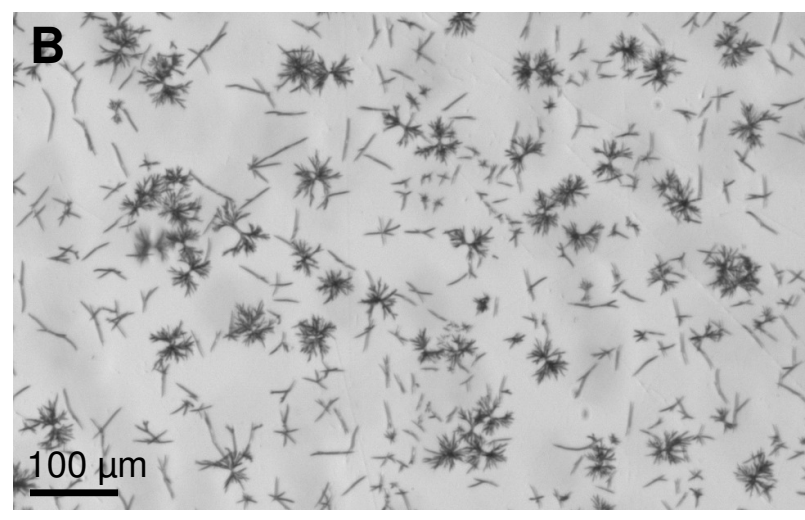

$\left[\mathrm{Na}_{2} \mathrm{CO}_{3}\right]_{n}=0.035 \quad\left[\mathrm{BaCl}_{2}\right]_{n}=0.06$ $\mathrm{Q}=0.1 \mathrm{~mL} / \mathrm{min}$

FIG. 3. Microscope (Nikon SMZ18) images showing the characteristic particle size and shape of $\mathrm{CaCO}_{3}(\mathrm{~A})$ and $\mathrm{BaCO}_{3}(\mathrm{~B})$ precipitates.

only a low impact on the fluid dynamics. They are advected by the flow and hence a circularly spreading $(d \approx 1)$ and homogeneously filled $(F \approx 1)$ precipitation pattern is obtained for both the $\mathrm{Ca}^{2+}$ and the $\mathrm{Ba}^{2+}$ systems (not shown here). We further note that the total grayscale intensity, $I_{\text {tot }}$, of the experimental images will be used to qualitatively measure the amount of precipitate produced for different reactant concentrations and flow rates. Even though the refractive indexes of the $\mathrm{CaCO}_{3}$ and $\mathrm{BaCO}_{3}$ particles are significantly different due to their different size and shape (Fig. 3), the trends are still comparable. Indeed, Fig. 4A shows that, injecting $\mathrm{CO}_{3}^{2-}$ solutions with increasing concentration into a low concentration $\mathrm{Ca}^{2+}$ solution at a low flow rate yields more and more precipitate i.e. $I_{\text {tot }}$ increases. This trend is also clearly recovered for the case of $\mathrm{Ba}^{2+}$ (Fig. 4B) even if the absolute values of $I_{\text {tot }}$ are not comparable with those for $\mathrm{CaCO}_{3}$.

For low reactant concentrations, the patterns are similar even for higher flow rates $Q$ (Fig. 2A1 and 2A2). They are mainly circular with a large $d$ value. The zigzagged shape of the inner periphery is caused by the strong flow flushing away the precipitate particles from the inlet region. The main difference between the two patterns is expressed in their $F$ values. The $\mathrm{CaCO}_{3}$ pattern with $F=0.68$ (Fig. 2A1) seems to be less hollow than the $\mathrm{BaCO}_{3}$ with $F=0.39$ (Fig. 2A2). This may be caused by the different particle shapes. The $\mathrm{CaCO}_{3}$ particles are small and separated thus they sediment once the liquid motion is not strong enough to carry them (Fig. 3A). This leads to the evolution of a thin (but detectable) precipitate layer behind the main rim of the pattern (Fig. 2A1). In comparison, the $\mathrm{BaCO}_{3}$ 

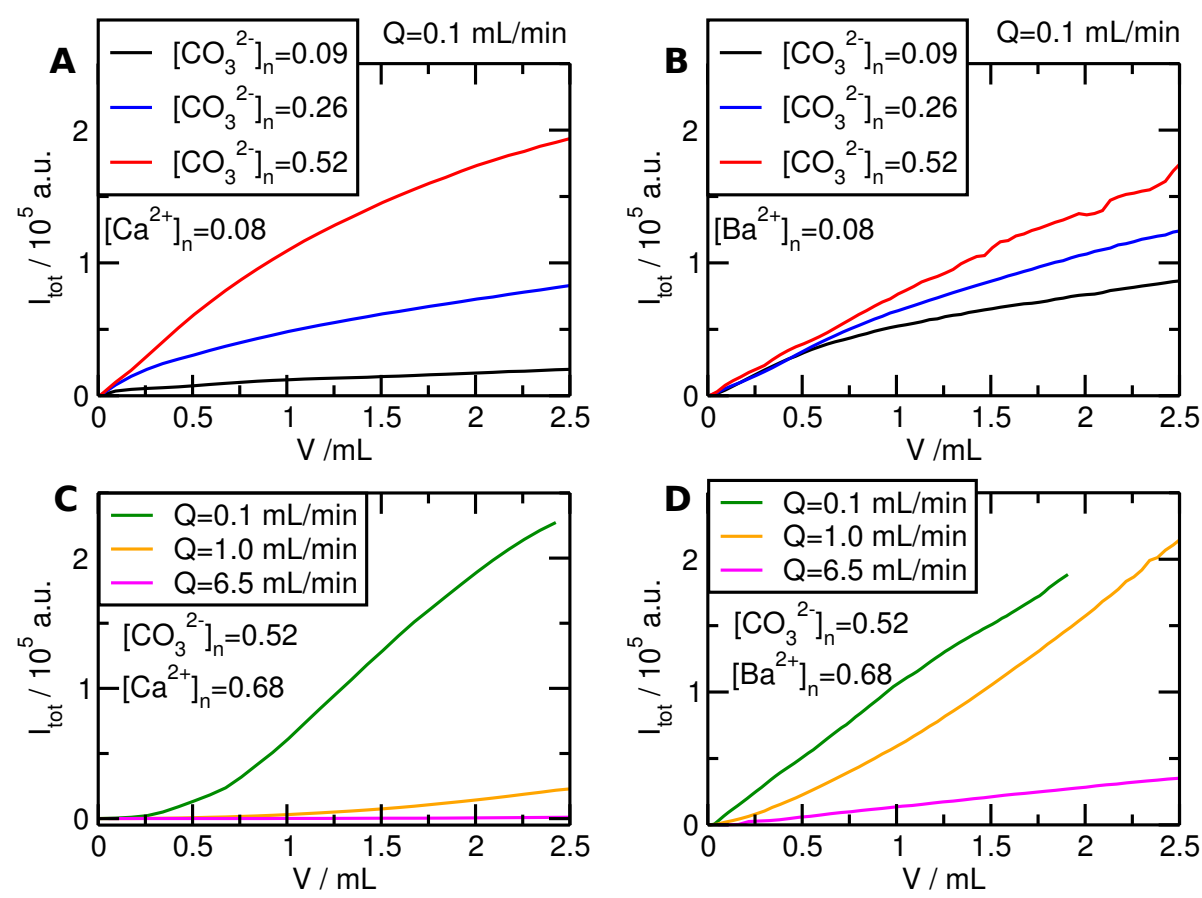

FIG. 4. Total grayscale intensity, $I_{\text {tot }}$, as a function of the injected volume of the $\mathrm{Na}_{2} \mathrm{CO}_{3}$ solution for various values of the experimental parameters. Panels $\mathrm{A}$ and $\mathrm{C}$ correspond to $\mathrm{CaCO}_{3}$ while panels $\mathrm{B}$ and $\mathrm{D}$ relate to $\mathrm{BaCO}_{3}$ precipitates.

needles have a tendency to stick together and form three-dimensional star-like structures (Fig. 3B). These structures collect other needles while they are drifted by the flow. The brushing behavior results in a smaller precipitate area behind the main rim of the pattern leading to lower $F$ values. However, apart from this difference in the interior region, $\mathrm{Ca}^{2+}$ and $\mathrm{Ba}^{2+}$ produce similar patterns for all flow rates at such low concentrations.

Fig. 2A3 and 4 show that increasing the concentration of the injected carbonate solution at low $\left[\mathrm{M}^{2+}\right]$ yields a more irregular fingered pattern. This is related to the fact that more precipitate is produced (Fig. 4A,B) and hence the permeability of the cell locally decreases. This provides conditions for a precipitation-driven instability due to the fact that the more mobile injected liquid locally pushes the less mobile solid phase ${ }^{22,23}$. Fig. $2 \mathrm{~A} 3$ and 4 show that the patterns emerging via this precipitation-driven fingering instability are similar in shapes and characterized by similar $F$ and $d$ values for both $\mathrm{Ca}^{2+}$ and $\mathrm{Ba}^{2+}$. The difference in the pattern texture coming from the shape of the precipitate particles is nevertheless visible again. The $\mathrm{CaCO}_{3}$ pattern exhibits smoother edges for the fingers (Fig. 2A3) while they are much more rigid and aggregated for $\mathrm{BaCO}_{3}$ (Fig. 2A4). This similarity between 
$\mathrm{CaCO}_{3}$ and $\mathrm{BaCO}_{3}$ patterns holds also for higher reactant concentrations provided $Q$ is not too large (Fig. 2A5, and A6). Experiments performed with those reactant concentrations yield a large amount of precipitate.

If both the reactant concentrations and flow rate $Q$ are further increased, hollow tube-like patterns emerge in both systems (Fig. 2A7 and A8). Such tube patterns appear because a large amount of precipitate is instantaneously produced across the gap in the small region where the reactants mix. This cohesive precipitate behaves like a physical barrier between the reactant solutions and hinders further mixing, leading to a sharp drop in the amount of precipitate further produced (Fig. 4C,D). Although both patterns feature this characteristic precipitate wall structure, there are nevertheless some differences. The $\mathrm{CaCO}_{3}$ pattern (Fig. 2A7) is hollow $(F=0.06)$ and not too elongated $(d=0.56)$ because the monodisperse $\mathrm{CaCO}_{3}$ particles (Fig. 3A) stick together forming a tough precipitate wall with low permeability across the entire gap width. Although we cannot perform in situ sample analysis in the sealed reactor, X-ray analysis of the particles collected after our experiment or of $\mathrm{CaCO}_{3}$ samples obtained in other similar experimental flow conditions ${ }^{9}$ show that they are composed of cubic shaped calcite. Their aggregate expands therefore more or less uniformly with a homogeneous permeability distribution along its periphery. In contrast, the $\mathrm{BaCO}_{3}$ pattern (Fig. 2A8) consists of elongated hollow precipitate channels (smaller $d$ ) along a zone with a denser precipitate arranged in curly and closed channels. This more compact zone induces a larger filling $F$ and appears because of the three-dimensional star-like shape of the $\mathrm{BaCO}_{3}$ particles (Fig. 3B) hindering the formation of a tough and uniform precipitate wall as in the case of calcium. Therefore, leakage of the reactant solutions between the $\mathrm{BaCO}_{3}$ loosely tight agglomerated particles leads to a further growth of side channels. Because those side channels are fed through small holes in the wall of the main channel, their inner diameter is smaller than the gap width. This induces precipitation all around the invading liquid parts producing secondary layered structures, similar to some three-dimensional chemical gardens grown upon injection ${ }^{24}$. The filling $F$ is therefore larger than in the calcium case. Despite these differences between the patterns of Fig. 2A7 and 2A8, their main characteristic, namely that a precipitate wall forms which significantly modifies the hydrodynamics, is common.

Fig. 2B illustrates the main differences between the $\mathrm{CaCO}_{3}$ and $\mathrm{BaCO}_{3}$ precipitation patterns obtained in some range of the experimental conditions. As shown recently ${ }^{2}$, a hollow $\mathrm{CaCO}_{3}$ precipitation pattern can be obtained if both reactants are highly concentrated and 

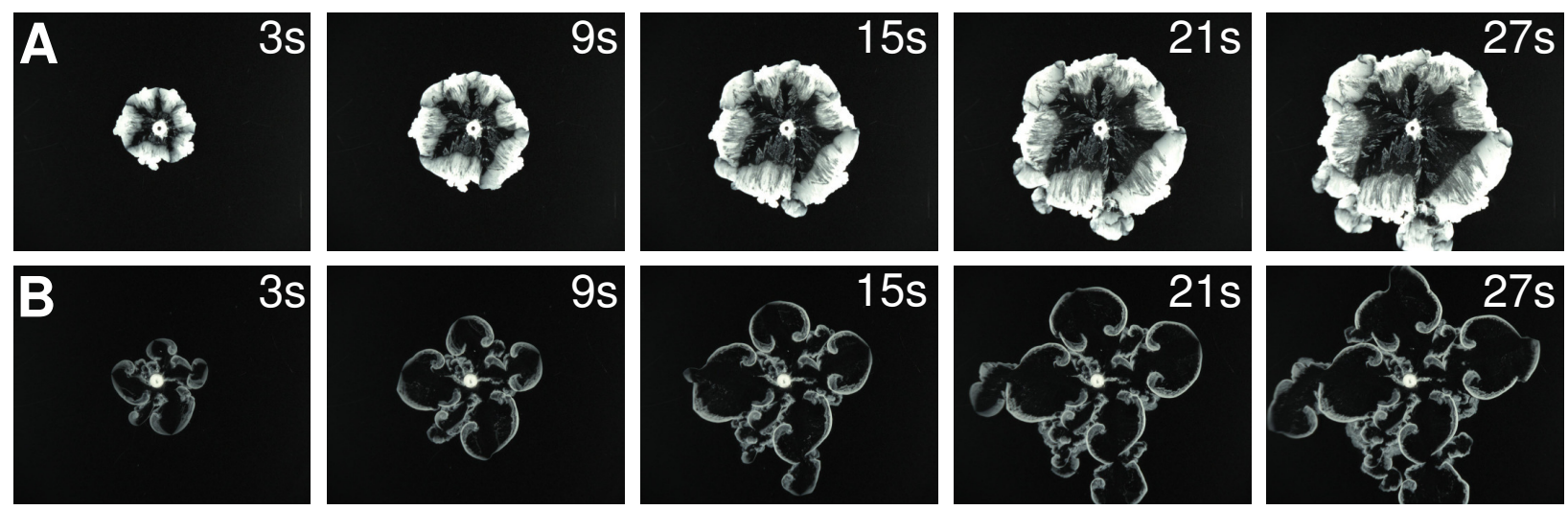

FIG. 5. Experimental images showing the time evolution of $\mathrm{CaCO}_{3}$ (panel A) and $\mathrm{BaCO}_{3}$ (panel B) patterns for identical experimental parameters $\left(\left[\mathrm{CO}_{3}^{2-}\right]_{n}=0.26,\left[\mathrm{M}^{2+}\right]_{n}=0.68, Q=6.5 \mathrm{~mL} / \mathrm{min}\right)$.

the flow rate is beyond a critical value. Fig. 2B1 and 2B2 point out that the critical flow rate for transition towards such hollow structures may depend on the reactants. The $\mathrm{CaCO}_{3}$ pattern emerging at medium flow rate (Fig. $2 \mathrm{~B} 1, Q=1.0 \mathrm{~mL} / \mathrm{min}$ ) is essentially hollow $(F=0.14)$ and grows in some preferred directions $(d=0.28)$. In contrast, the $\mathrm{BaCO}_{3}$ pattern is almost fully filled with precipitate (Fig. 2B2, $F=0.96$ ) and is more circular $(d=0.61)$. The $\mathrm{BaCO}_{3}$ precipitate is less compact due to the three-dimensional star-like shape of the solid particules, thus the invading reactant can pass through the precipitate layer and react.

Other differences between the patterns are seen on Fig. 2 B3 and 4, when $Q$ is large but the concentration of the metal ion solution is decreased. While those experimental conditions still provide a hollow pattern for $\mathrm{CaCO}_{3}$, the $\mathrm{BaCO}_{3}$ system is full of small broken spirals. This shows that the $\mathrm{Ba}^{2+}$ concentration is here again not high enough to produce a precipitate wall that can sustain the interior pressure while pumping is continued.

Finally, for reactant concentrations for which similar patterns were found at $Q=$ $1.0 \mathrm{~mL} / \mathrm{min}$ in both systems (Fig. 2A5,6), a larger $Q$ results in essentially different patterns (Fig. 2B5,6). In the case of $\mathrm{CaCO}_{3}$, the $\mathrm{CO}_{3}^{2-}$ concentration is not high enough to produce a tough wall with low permeability, thus the precipitate wall thickens with time. However, the wall is strong enough to move as a whole without breaking, leading to an expanding wall structure as shown in the temporal evolution featured in Fig. 5A. For identical values of the experimental parameters, $\mathrm{BaCO}_{3}$ exhibits a much less filled precipitation pattern (Fig. 2B6) with well visible spirals similar to those described recently in chemical gardens ${ }^{1}$. For those 
spirals to evolve, it is crucial to form a circular precipitate wall during the radial spreading ${ }^{1}$. If that wall is not strong enough and breaks at some point, further injection rotates parts of the precipitate wall around the breaking point leading to the growth of spirals. The rotation of the wall pieces and the evolution of spirals may be followed in time in Fig. 5B. The presence of those spirals in the $\mathrm{BaCO}_{3}$ system and their absence in the $\mathrm{CaCO}_{3}$ system indicate again that a stronger and more cohesive precipitate is produced if $\mathrm{Ca}^{2+}$ and $\mathrm{CO}_{3}^{2-}$ reactants are used.

\section{DISCUSSION}

We have experimentally investigated whether two different precipitation reactions performed in a confined geometry upon radial injection of a solution of carbonate into a metal ion solution may lead to similar patterns or not. The alkaline earth metal ions used here $\left(\mathrm{Ca}^{2+}\right.$ and $\left.\mathrm{Ba}^{2+}\right)$ may show similar tendency to form carbonate precipitates as far as the experimental parameters are similar. However, the precipitation-driven pattern formation depends not only on the chemical properties of the reactant solutions but also on the properties of the solid product.

Similar patterns are obtained when both the carbonate and the metal ions are in low concentrations (Fig. 2A1 and 2A2). In that case, the precipitate particles are much smaller than the gap width and do not form aggregates. As a consequence, the precipitate more or less passively follows the fluid flow. However, a major interplay between precipitation and fluid dynamics is obtained when the size of the precipitate particles or of their aggregates becomes comparable to the characteristic length of the confinement. This is the case for the experimental conditions leading to the formation of hollow tube-like patterns (Fig. 2A7 and $2 \mathrm{~A} 8)$.

Similar patterns may be obtained using higher reactant concentrations and/or flow rate provided both reactant systems are in conditions beneath (Fig. 2A5,6) or beyond (Fig. 2A7,8) the critical values of parameters required for tube formation. However, those critical parameter values depend on the physico-chemical properties of the reactants and of the products. Thus, a same set of parameter values may be simultaneously supercritical for one reaction but subcritical for the other one (Fig. 2B1,2,3,4). This is illustrated for an idealized case in Fig. 6. The parameter values inside the dark prism correspond to such reactant concentra- 
tions and flow rates where no tube formation is obtained in the $\mathrm{Ca}^{2+}+\mathrm{CO}_{3}^{2-}$ reaction (i.e. the system is subcritical). Though the surface of the dark prism corresponding to the sets of critical parameters is shown in the sketch as being abrupt, the transition from subcritical to supercritical state may be smooth in experiments. The same analogy is used for the interior and exterior parts of the pale prism corresponding to the subcritical and supercritical states of the $\mathrm{Ba}^{2+}+\mathrm{CO}_{3}^{2-}$ system, respectively. Therefore, performing experiments for values of parameters inside the dark prism (i.e. subcritical for both $\mathrm{Ba}^{2+}$ and $\mathrm{Ca}^{2+}$ systems with low reactant concentrations and flow rates) or outside the pale prism (supercritical for both systems with large reactant concentrations and flow rates) results in similar $\mathrm{CaCO}_{3}$ and $\mathrm{BaCO}_{3}$ patterns. On the contrary, when one reactant pair is subcritical and the other one is supercritical (i.e. within the pale prism but outside the dark prism in Fig. 6), or when both of them are in the transient regime close to the critical values, quite different precipitation patterns can be observed between $\mathrm{CaCO}_{3}$ and $\mathrm{BaCO}_{3}$ for same values of parameters (Fig. 2B).

Hence, in a real porous media flow, when the particle size is comparable to the pore size of the medium, the difference shown here on the example case of $\mathrm{CaCO}_{3}$ and $\mathrm{BaCO}_{3}$ particles may lead to significantly different precipitation behaviors. Therefore, in experiments aiming to understand underground precipitation, care has to be taken when the model system is chosen.

\section{CONCLUSION}

We have experimentally investigated precipitation-driven pattern formation upon radial injection in a confined geometry using the $\mathrm{Ca}^{2+}+\mathrm{CO}_{3}^{2-}$ and $\mathrm{Ba}^{2+}+\mathrm{CO}_{3}^{2-}$ reactant pairs. It has been shown that replacing the $\mathrm{Ca}^{2+}$ by $\mathrm{Ba}^{2+}$ ions can produce similar but also different patterns depending on the experimental values of parameters. For conditions where tubes form, the size of the individual particles or of their aggregates is comparable to the size of the confinement. If both reaction pairs are then in concentrations or with a flow rate beneath or beyond those critical values, similar patterns are found. However, some differences are also visible due to the shape and size of the solid particules. Different patterns are observed when one of the reactant pair precipitates for conditions beneath these critical values while the other one precipitates beyond them, or when both of them are in the transient regime. 


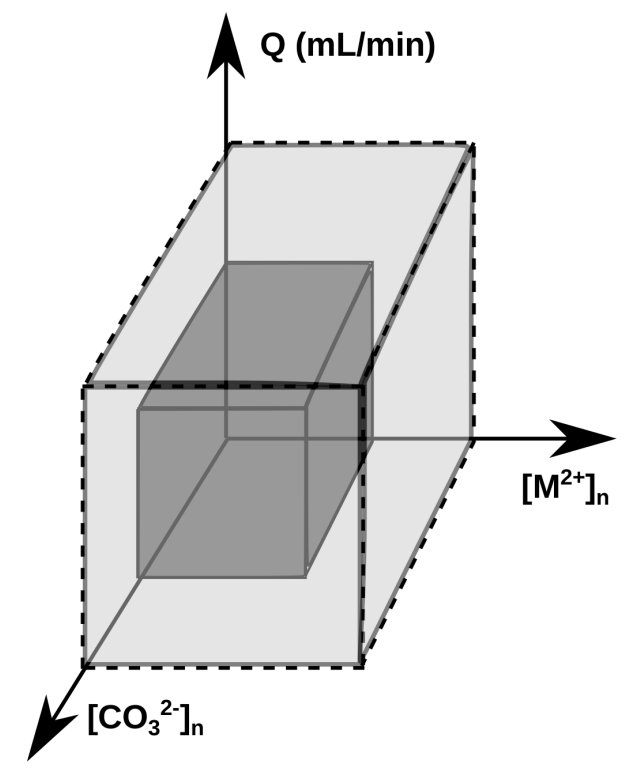

FIG. 6. Schematic of the parameter space illustrating the different set of experimental parameter values required for $\mathrm{Ca}^{2+}+\mathrm{CO}_{3}^{2-}$ (inside dark rectangular prism) and $\mathrm{Ba}^{2+}+\mathrm{CO}_{3}^{2-}$ (outside pale rectangular prism) systems to produce tube-like patterns.

Therefore, from a pattern formation point of view, the reactants of a precipitation reaction can be replaced by a chemically similar system provided suitable experimental parameters are chosen.

\section{ACKNOWLEDGMENTS}

We thank F. Brau and M. Schröter for fruitful discussions as well as Prodex for financial support.

\section{REFERENCES}

\section{REFERENCES}

${ }^{1}$ Haudin, F., J. H. E. Cartwright, F. Brau, and A. De Wit (2014), Spiral Precipitation Patterns in Confined Chemical Gardens, Proc. Nat. Acad. Sci. (USA), 111, 17363-17367.

${ }^{2}$ Schuszter, G., F. Brau, and A. De Wit (2016), Calcium Carbonate Mineralization in a Confined Geometry, Environ. Sci. Technol. Lett., 3(4), 156-159. 
${ }^{3}$ Bohner, B., G. Schuszter, O. Berkesi, D. Horváth, and Á. Tóth (2014), Self-organization of Calcium Oxalate by Flow-driven Precipitation, Chem. Commun., 50, 4289.

${ }^{4}$ Makki, R., L. Roszol, J.J. Pagano, and O. Steinbock (2012), Tubular Precipitation Structures: Materials Synthesis Under Non-equilibrium Conditions, Phil. Trans. R. Soc., 370, $2848-2865$.

${ }^{5}$ Barge, L. M., S. S. S. Cardoso, J. H. E. Cartwright, G. J. T. Cooper, L. Cronin, A. De Wit, I. J. Doloboff, B. Escribano, R. E. Goldstein, F. Haudin, D. E. H. Jones, A. L. Mackay, J. Maselko, J. J. Pagano, J. Pantaleone, M. J. Russell, C. I. Sainz-Daz, O. Steinbock, D. A. Stone, Y. Tanimoto, and N. L. Thomas (2015), From Chemical Gardens to Chemobrionics, Chem. Rev., 115(16), 8652-8703.

${ }^{6}$ Roszol, L., and O. Steinbock (2011), Controlling the Wall Thickness and Composition of Hollow Precipitation Tubes, Phys. Chem. Chem. Phys., 13, 20100-20103.

${ }^{7}$ Haudin, F., J.H.E. Cartwright, and A. De Wit (2015), Direct and Reverse Chemical Garden Patterns Grown upon Injection in Confined Geometries, J. Chem. Phys. C, 119, $15067-15076$.

${ }^{8}$ Pusztai, P., E. Tóth-Szeles, D. Horváth, Á. Tóth, Á. Kukovecz, and Z. Kónya (2016), A Simple Method to Control the Formation of Cerium Phosphate Architectures, CrystEngComm, 17, 8477.

${ }^{9}$ Bohner, B., G. Schuszter, D. Horváth, and Á. Tóth (2015), Morphology Control by Flowdriven Self-organizing Precipitation, Chem. Phys. Lett., 631-632, 114-117.

${ }^{10}$ Bohner, B., B. Endrődi, D. Horváth, and Á. Tóth (2016), Flow-driven Pattern Formation in the Calcium-oxalate System, J. Chem. Phys., 144, 164504.

${ }^{11}$ Tóth-Szeles, E., G. Schuszter, Á. Tóth, Z. Kónya and D. Horváth (2016), Flow-driven Morphology Control in the Cobalt-Oxalate System, CrystEngComm, 18, 2057-2064.

${ }^{12}$ Metz, B., O. Davidson, H.C. de Conninck, M. Loos, and L.A. Meyer, Eds. IPCC Special Report on Carbon Dioxide Capture and Storage; Cambridge University Press: New-York, 2005.

${ }^{13}$ Matter, J. M., M. Stute, S. Ó. Snæbjörnsdottir, E. H. Oelkers, S. R. Gislason, E. S. Aradottir, B. Sigfusson, I. Gunnarsson, H. Sigurdardottir, E. Gunnlaugsson, G. Axelsson, H. A. Alfredsson, D. Wolff-Boenisch, K. Mesfin, D. F. de la Reguera Taya, J. Hall, K. Dideriksen, and W. S. Broecker (2016), Rapid Carbon Mineralization for Permanent Disposal of Anthropogenic Carbon Dioxide Emissions, Science, 352(6291), 1312-1314. 
${ }^{14}$ Jean, J.-S, H.-I. Hsiang, Z. Li, C.-L. Wong, H.-J. Yang, K.-M. Yang, C.-L. Wang, H.-W. Lin, and C.-C. Kuo (2016), Influence of Supercritical $\mathrm{CO}_{2}$ on the Mobility and Desorption of Trace Elements from $\mathrm{CO}_{2}$ Storage Rock Sandstone and Caprock Shale in a Potential $\mathrm{CO}_{2}$ Sequestration Site in Taiwan, Aerosol and Air Quality Research, 16(7), 1730-1741.

${ }^{15}$ Luquot, L. and P. Gouze (2009), Experimental Determination of Porosity and Permeability Changes Induced by Injection of $\mathrm{CO}_{2}$ into Carbonate Rocks, Chem. Geo., 265, $148-159$.

${ }^{16}$ Hubbell, J.H. and S.M. Seltzer (1989), http://www.nist.gov/pml/data/xraycoef/, NIST Physical Measurement Laboratory.

${ }^{17}$ Carmona-Quiroga, P.M. and M.T. Blanco-Varela (2013), Ettringite Decomposition in the Presence of Barium Carbonate, Cement and Concrete Research, 52, 140-148.

${ }^{18}$ Zezulová, A., T. Stanĕk, and T. Opravil (2016), The Influence of Barium Sulphate and Barium Carbonate on the Portland Cement, Procedia Engineering, 151, 42-49.

${ }^{19}$ Park, S., J.-H. Bang, K. Song, C.W. Jeon, and J. Park (2016), Barium Carbonate Precipitation as a Method to Fix and Utilize Carbon Dioxide, Chemical Engineering Journal, 284, 1251-1258.

${ }^{20}$ Haudin, F., V. Brasiliense, J.H.E. Cartwright, F. Brau, and A. De Wit (2015), Genericity of Confined Chemical Garden Patterns with Regard to Changes in the Reactants, Phys.Chem.Chem.Phys., 17, 12804.

${ }^{21}$ Schuszter, G., F. Brau, and A. De Wit (2016), Flow-driven Control of Calcium Carbonate Precipitation Patterns in a Confined Geometry, Phys. Chem. Chem. Phys., 18, 25592.

${ }^{22}$ Nagatsu, Y., Y. Ishii, Y. Tada, and A. De Wit (2014), Hydrodynamic Fingering Instability Induced by a Precipitation Reaction, Phys. Rev. Lett, 113, 024502.

${ }^{23}$ Shukla, P. and A. De Wit (2016), Fingering Dynamics Driven by a Precipitation Reaction: Nonlinear Simulations, Phys. Rev. E, 93, 023103.

${ }^{24}$ Thouvenel-Romans, S., and O. Steinbock (2003), Oscillatory Growth of Silica Tubes in Chemical Gardens, J. Am. Chem. Soc., 125(14), 4338-4341. 